\title{
EDITORIAL
}

\section{Triple therapy in idiopathic pulmonary fibrosis: an alarming press release}

\author{
A.U. Wells*, J. Behr ${ }^{\#, \uparrow}$, U. Costabel ${ }^{+, \S}$, V. Cottin ${ }^{\dagger}$ and V. Poletti**
}

0 n October 21, 2011, the National Heart, Lung, and Blood Institute (NHLBI), announced by press release that the prednisolone/azathioprine/ $N$-acetylcysteine (triple therapy) arm of the PANTHER study (Prednisone, Azathioprine and N-acetylcysteine: a Study That Evaluates Response in IPF) had been discontinued [1]. Compared with patients receiving placebo, patients with idiopathic pulmonary fibrosis (IPF) treated with triple therapy had excess deaths ( $11 \%$ versus $1 \%)$, more hospitalisations ( $29 \%$ versus $8 \%$ ) and a higher prevalence of serious adverse events ( $31 \%$ versus $9 \%$ ). The study is continuing with the $N$-acetylcysteine and placebo arms only. No concerns have been raised as a result of preliminary analysis of the $\mathrm{N}$-acetylcysteine monotherapy arm of the PANTHER study. The preliminary announcement by the NHLBI has no immediate implications with regard to the introduction or continuation of $\mathrm{N}$-acetylcysteine in IPF.

It is hardly surprising that this announcement has caused widespread alarm amongst medical practitioners and patients with IPF currently taking triple therapy, especially in Europe, where this therapy is largely used. In isolation, the small absolute number of excess deaths in the triple therapy arm, although statistically significant, could not be viewed as conclusive. In earlier IPF studies, the supposed efficacy of interferon- $\gamma$ [2] and the protective effect of pirfenidone against acute exacerbations [3] were based on similarly low numbers of events and neither finding was confirmed in subsequent larger studies. However, the current findings differ in one essential respect: the increased mortality in the triple therapy arm was associated with a conclusive excess of hospitalisations and serious adverse events.

In an earlier US trial of interferon- $\gamma$, hospital admissions were largely ascribable to infections and acute exacerbations [4]. If the same pattern emerges on detailed analysis of the triple therapy arm of the PANTHER study, it will be essential to explore whether the adverse outcome can be attributed to the complications of immunosuppressive therapy in elderly patients, given the fact that almost half of the enrolled patients were aged $>70 \mathrm{yrs}$. It is not yet clear whether the excess

\footnotetext{
*Interstitial Lung Disease Unit, Royal Brompton Hospital, London, UK. "Internal Medicine I Pulmonary Division, University of Munich, Munich, "Dept of Internal Medicine III - Respiratory and Critical Care, Ruhr-University Bochum, Bochum, ${ }^{+}$Medical Faculty, University of Duisburg-Essen, and ${ }^{\S}$ Ruhrlandklinik, Essen, Germany. ${ }^{f}$ Service de Pneumologie, Hôpital Louis Pradel, Lyon, France. **Pierantoni-Morgagni Hospital, Forlì, Italy.
}

CORRESPONDENCE: A.U. Wells, Interstitial Lung Disease Unit, Royal Brompton Hospital, Emmanue Kaye Building, Manresa Road, London, SW3 6LR, UK. E-mail: athol.wells@rbht.nhs.uk morbidity and mortality in the triple therapy arm of the PANTHER study was due to acute exacerbations or infection, but this explanation would be compatible with the general clinical consensus that elderly patients are relatively more susceptible to the adverse immunosuppressive effects of azathioprine and more susceptible to respiratory infections.

These and other speculations, based on a press release, cannot be used to generate formal recommendations. Some uncertainties are likely to be resolved with the release of further analyses of patient data from the discontinued triple therapy arm. In particular, the individual characteristics (phenotype) of the IPF patients enrolled in this study, and how they compare to past study populations and to IPF patients seen in routine clinical practice, is not yet known. However, this does not alter the fact that clinicians and patients urgently require guidance as to the immediate implications of these findings in routine practice. That guidance can only take the form of a statement of how the findings are likely to be viewed by the majority of patients and clinicians. This has prompted us to provide a consensus view, based on widespread informal discussion amongst delegates at a large recent European IPF meeting.

The strong recommendation made by the authors is that the press release should be proactively discussed with all patients with definite or probable IPF, currently receiving immunosuppressive therapy, in order to make a shared decision on subsequent management. It is for this reason that we strongly recommend that the press release should be discussed with all patients with definite or probable IPF receiving immunosuppressive therapy. Over and above a review of the press release itself, informed discussion should include a summary of the aforementioned uncertainties and detailed consideration of the specific clinical scenario of the individual patient. We recommend strongly that the patient should be offered the opportunity to take a view on management changes and, in clinical scenarios in which management decisions are otherwise a close call, strongly held views of the patient should determine the final management decision.

It should be stressed that it is entirely unclear whether the reported adverse findings are specific to the use of azathioprine or to the combination of azathioprine and low-dose steroid therapy, or apply to the use of immunosuppressive therapy at large in IPF. It is for this reason that we strongly recommended that the press release should be discussed with all patients with definite or probable IPF receiving immunosuppressive therapy. We anticipate that, pending further analyses, most patients and physicians will decide against starting immunosuppressive 
therapy de novo in IPF. Similarly, most patients and clinicians are likely to withdraw immunosuppressive therapy if disease is continuing to progress despite treatment. However, clinical decisions will be less straightforward in other patients already receiving immunosuppressive therapy and the following scenarios have therefore been explored.

1) In patients with definite IPF already established on triple therapy or other immunosuppressive therapy for $\geqslant 12$ months, with no evidence of progression of IPF and no major complications from treatment, we caution against the precipitous withdrawal of immunosuppressive therapy. We believe that most patients and clinicians will choose to continue treatment in this setting, given the extreme desirability of maintaining disease stability, once achieved.

2) In patients with definite IPF receiving triple therapy or other immunosuppressive therapy for $<12$ months, clinical decisions can only be made on a case-by-case basis. It is likely that the majority of patients and clinicians will choose to discontinue immunosuppressive therapy based on the press release if treatment has been in place for $<6$ months, but if treatment has been well tolerated for over 6 months, decisions will be finely balanced.

3) In patients with definite IPF, with significant infective complications from triple therapy or other immunosuppressive therapy, whether early in the course of treatment or subsequently, we anticipate that most patients and clinicians will choose to discontinue immunosuppressive therapy.

4) In patients with probable or possible IPF, but a realistic differential diagnosis of chronic hypersensitivity pneumonitis, fibrotic nonspecific interstitial pneumonia or another progressive fibrotic disorder, we caution strongly against the precipitous withdrawal of triple therapy or other forms of immunosuppressive therapy. Decisions can only be made on a case-by-case basis, taking into account the likelihood of IPF but also the fact that a good long-term outcome is unlikely in IPF but can be achieved by optimal treatment of non-IPF fibrotic lung disease.

5) It should be stressed that the press release is not relevant to patients with definite nonspecific interstitial pneumonia, fibrotic hypersensitivity pneumonitis, connective tissue disease-associated pulmonary fibrosis or other scenarios in which IPF is not a realistic diagnosis.

\section{STATEMENT OF INTEREST}

Statements of interest for A.U. Wells, J. Behr, U. Costabel and V. Cottin can be found at www.erj.ersjournals.com/site/misc/statements.xhtml

\section{REFERENCES}

1 National Heart, Lung and Blood Institute. Commonly used threedrug regimen for idiopathic pulmonary fibrosis found harmful. www.nih.gov/news/health/oct2011/nhlbi-21.htm Date last accessed: November 2, 2011. Date last updated: October 21, 2011.

2 Ziesche R, Hofbauer E, Wittmann $\mathrm{K}$, et al. A preliminary study of long-term treatment with interferon gamma- $1 \mathrm{~b}$ and low-dose prednisolone in patients with idiopathic pulmonary fibrosis. N Engl J Med 1999; 341: 1264-1269.

3 Azuma A, Nukiwa T, Tsuboi E, et al. Double-blind, placebocontrolled trial of pirfenidone in patients with idiopathic pulmonary fibrosis. Am J Respir Crit Care Med 2005; 171: 1040-1047.

4 Martinez FJ, Safrin S, Weycker D, et al. The clinical course of patients with idiopathic pulmonary fibrosis. Ann Intern Med 2005; 142: 963-967. 